\title{
"Adolphe" de Benjamin Constant. Postérité d'un roman (1816-2016), dir. L. BURNAND et G. POISSON
}

\section{Aline Hodroge}

\section{(2) OpenEdition}

\section{Journals}

\section{Édition électronique}

URL : https://journals.openedition.org/studifrancesi/31828

DOI : 10.4000/studifrancesi.31828

ISSN : 2427-5856

Éditeur

Rosenberg \& Sellier

\section{Édition imprimée}

Date de publication : 1 août 2020

Pagination : 410-411

ISSN : 0039-2944

\section{Référence électronique}

Aline Hodroge, " "Adolphe" de Benjamin Constant. Postérité d'un roman (1816-2016), dir. L. BuRnand et G. poIsson », Studi Francesi [En ligne], 191 (LXIV | II) | 2020, mis en ligne le 01 septembre 2020, consulté le 18 septembre 2021. URL : http://journals.openedition.org/studifrancesi/31828 ; DOI : https://doi.org/ 10.4000/studifrancesi.31828

Ce document a été généré automatiquement le 18 septembre 2021.

\section{(†)

Studi Francesi è distribuita con Licenza Creative Commons Attribuzione - Non commerciale - Non opere derivate 4.0 Internazionale. 


\title{
"Adolphe" de Benjamin Constant. Postérité d'un roman (1816-2016), dir. L. BURNAND et G. POISSON
}

\author{
Aline Hodroge
}

\section{RÉFÉRENCE}

“Adolphe” de Benjamin Constant. Postérité d'un roman (1816-2016), dir. L. BURNAND et G. PoIsson, Genève, Slatkine, 2016, 160 pp.

1 À l'occasion des deux cents ans de la parution d'Adolphe de Benjamin Constant, Léonard Burnand et Guillaume Poisson ont dirigé un ouvrage qui décline les multiples facettes de ce roman. Publié en 1816, Adolphe suscite de nombreuses réactions chez son lectorat, aussi bien contemporain qu'actuel. Pourquoi ce court roman, mettant en scène les amours d'Adolphe et d'Ellénore, ne cesse-t-il d'engendrer les rééditions et les relectures? Cet ouvrage décrypte ainsi le rayonnement de ce texte à travers quatre axes, mettant à contribution plusieurs spécialistes de l'œuvre constantienne. La première partie de ce volume se construit autour du texte romanesque et de son parcours éditorial («Éditer Adolphe»); la deuxième s'intéresse à sa transposition dans d'autres langues («Traduire Adolphe»); la troisième poursuit cette transformation du texte à travers les différentes réécritures («Réécrire Adolphe») tandis que la quatrième partie («Adapter Adolphe») se concentre sur l'appropriation faite par d'autres médias tels que le théâtre, le cinéma ou la bande dessinée.

2 Cet ouvrage s'ouvre sur un avant-propos rédigé par le directeur de l'Institut Benjamin Constant. Léonard Burnand et Guillaume Poisson ont ponctué l'année 2016 d'événements autour d'Adolphe en proposant, notamment, une exposition à la Bibliothèque cantonale et universitaire de Lausanne (site Riponne). En effet, c'est elle qui réunit aujourd'hui le plus grand fonds consacré à l'auteur lausannois. La première partie, sur l'édition d'Adolphe, s'ouvre ainsi sur l'exploitation de ce fonds par plusieurs 
chercheurs. Paul DELBOUILLE (Préhistoire et publication du roman du vivant de son auteur, pp.13-19) revient sur les biographèmes susceptibles d'avoir influencé l'écriture de Constant: Charlotte Du Tertre et Germaine de Staël ont ainsi marqué durablement la vie de l'auteur. Paul Delbouille analyse ensuite les manuscrits, conservés aujourd'hui à la BnF et à la BCUL, à partir desquels il est possible de suivre l'histoire éditoriale d'Adolphe. Cet article, magnifiquement illustré par des photographies de ces documents, fait état de deux éditions, dès 1816, tandis que deux autres suivront en 1824 et en 1828 . Guillaume PoIsson (Les éditions illustrées d'“Adolphe", pp. 21-32) poursuit l'enquête en mettant à profit de nombreuses illustrations du roman constantien, depuis ses débuts (1847) jusqu'au $\mathrm{xx}^{e}$ siècle où ce phénomène se généralise. Plusieurs scènes-clefs reviennent sous le crayon des illustrateurs, chacun choisissant de mettre en avant les paysages, le cadre temporel du roman ou un trait caractéristique d'un personnage. Frédéric Régamey, Georges Jeanniot ou encore Serge de Solomko sont ainsi mis à l'honneur, sans compter la double page consacrée au travail de René-Victor Auberjonois. Paul DelBouille (Les éditions critiques d'“Adolphe”, pp. 33-39) reprend la plume pour clore cette première partie sur les différentes éditions critiques qui se sont succédé au $\mathrm{xx}^{\mathrm{e}}$ siècle. Chacune doit s'appuyer sur un texte et le choix est loin d'être simple: le texte de l'édition anglaise de 1816 séduit Gustave Rudler et Cecil Courtney tandis que Paul Delbouille favorise la première copie annotée de l'auteur, accessible à partir de fin 1973.

3 Le texte de Benjamin Constant ne se limite pas à la langue française: très tôt, il fait l'objet de traductions, sujet de cette deuxième partie. Cecil Courtney et Paul RowE (Alexandre Walker et la première traduction d"'Adolphe", pp. 43-49) s'intéressent à la traduction anglaise, proposée dès 1816 . Constant a même pu intervenir directement et conseiller Alexandre Walker. Cette entreprise ne sera renouvelée qu'en 1924 grâce à l'impulsion donnée par l'édition de Gustave Rudler. Eveline PASSET ("Adolphe" en allemand: le deux-points et le non-dit, pp. 51-54) soulève un questionnement autour des deux-points, difficiles à rendre dans la langue allemande. La première traduction date de 1817 , suivie par d'autres au $\mathrm{xx}^{\mathrm{e}}$ siècle: très peu respectent cette construction qui rend pourtant compte «d'une tension sémantique restant dans le non-dit» (p. 54). Adolphe connait un public toujours plus élargi avec sa traduction espagnole: WenceslaoCarlos Lozano ("Adolphe" en espagnol, pp. 57-59) fait état du vieillissement des traductions, dont la première date de 1828. Si le texte original ne rencontre pas ce problème, les nouvelles traductions sont essentielles afin de s'adapter au lectorat. Ilona KovÁcs ("Adolphe", versions hongroises, pp.61-65), quant à elle, analyse les trois traductions hongroises du texte de Constant. Les deux dernières contributions de cette partie dépassent les frontières européennes: Eijiro oHNO s'intéresse au rapport entretenu entre Adolphe et le public japonais ("Adolphe" au Japon, pp. 67-71): la forme convient ainsi «au goût préexistant des Japonais pour les textes intimes» (pp. 66-67) tandis que Minou MoshiRI (“Adolphe" en persan, pp. 73-75) décrit l'entreprise éditoriale surprenante d'un texte qui ne connait sa première traduction iranienne qu'en 2009. Chacune de ces traductions est accompagnée de plusieurs illustrations des éditions en question, montrant ainsi la manière dont chaque pays s'approprie Adolphe.

Cette appropriation de l'œuvre passe également par la réécriture et occupe la troisième partie de cet ouvrage. Gérard GENGEMBRE (Une réécriture «réaliste» d"'Adolphe". "La Muse du département" de Balzac, pp. 79-81) analyse la correspondance et le roman de Balzac afin de montrer comment le roman constantien alimente une réflexion autour d'un «idéal 
androgène ou hermaphrodite» (p. 81). Silvia LoRusso ("Ellénore" de Sophie Gay. Une réécriture sentimentale d"'Adolphe", pp. 83-87) s'intéresse au cas de Sophie Gay qui entend donner une autre version de l'histoire et prendre la défense d'Ellénore. Doris JAKUBEC (D'“Adolphe" à "Montclar", pp. 89-91), quant à elle, voit des affinités entre les années 1920 et la période Constant. Plus précisément, Pourtalès construit son roman par rapport à Adolphe, notamment dans sa conception du sentiment amoureux. Toujours dans une perspective chronologique, François RosSET (Rodolphe de $C^{* *}$, un grand blond tirant sur le roux, pp. 93-95) s'intéresse à la reprise de plusieurs motifs d'Adolphe dans La Polonaise de Stanislas d'Otremont. En tentant de donner la parole à Ellénore, l'auteur multiplie les sentences et s'attache à un conservatisme déplacé: «il y a méprise idéologique, esthétique et historique» (p.95). Donner la parole à Ellénore constitue un leitmotiv puisqu'Anne Boutin analyse, à son tour, Le point de vue d'Ellénore d'Ève Gonin (Et si Ellénore avait parlé..., pp.97-101). À l'aide d'un nouveau personnage-le prêtre confident-l'auteur donne un autre aperçu du rapport entre Adolphe et Ellénore. Surtout, cette entreprise souligne le choix romanesque de Constant et ses conséquences sur le lectorat. Béatrice finK (Facettes d'“Adolphe" dans "Providence" d'Anita Broockner. Une singulière mutation, pp. 103-105) s'intéresse à un roman passé inaperçu. Celui-ci cite Adolphe régulièrement tout en transposant son contenu dans l'intrigue. Roger FRANCILLON ( Une recréation moderne d'“Adolphe". "L'Imitation" de Jacques Chessex, pp. 107-111) analyse le «jeu de miroir» (p. 111) entre Jacques-Adolphe - protagoniste de L'Imitation - et Constant. En effet, Chessex s'inspire plutôt de l'auteur d'Adolphe en reprenant des biographèmes précis. Pour clore cette troisième partie, Adrienne ANGELO (Ellénore, c'est moi. Camille Laurens réécrit "Adolphe", pp. 113-117) interroge le lien qui unit le texte de Constant à Camille Laurens. Un véritable «jumelage auteur/personnage» (p. 113) se met en place et un dialogue s'installe entre les deux auteurs.

Ce dialogue se poursuit au-delà de l'écriture romanesque puisque Frédéric JAUNIN note plusieurs tentatives d'adaptations théâtrales ("Adolphe", l'impossible transposition scénique?, pp. 121-125). Malgré plusieurs tentatives, il semble difficile de proposer cette transposition en raison de la «rareté des dialogues directs dans le roman» (p. 125). L'adaptation du roman réussit mieux au cinéma: Antoine de BAECQUE ("Adolphe", un défi cinématographique, pp.127-131) retranscrit un entretien d'Isabelle Adjani, Chantal Thomas et Benoît Jacquot autour du film Adolphe, réalisé en 2002. Jan BAETENS, quant à lui, explore un tout autre média: la bande dessinée ("Adolphe" en bande dessinée, pp. 133-137). Il s'agit alors de trouver le juste équilibre entre le respect de l'œuvre et la nécessité de s'adapter à cette discipline. Dès lors, Adolphe s'inscrit dans «une nouvelle logique visuelle» (p. 134). L'ensemble du volume alterne les textes et les photographies, soigneusement choisies, afin de montrer la «postérité» d'Adolphe, tant au niveau de l'écriture que de l'illustration. Une Coda, présentée par Silvio CORSINI (Quand "Adolphe" inspire les créateurs contemporains, pp.141-153) réunit plusieurs créations uniques réalisées en 2015. Elles donnent alors à voir l'œuvre de Constant à travers différents matériaux.

6 Cet ouvrage ne pouvait mieux célébrer Adolphe de Constant: deux cents ans après sa parution, ce texte continue d'interpeller les chercheurs et suscite toujours un réel intérêt auprès du public francophone et étranger. La richesse des matériaux montre comment une œuvre parvient à survivre au temps: les rééditions, les emprunts, les réécritures et les adaptations assurent ainsi à l'auteur une "postérité», sans cesse renouvelée. 